\title{
A randomised control trial of early enteral nutrition $v$. conventional management in patients undergoing major resection for upper gastrointestinal cancer: a comparison of fluid management
}

\author{
R. C. Barlow and M. C. A. Puntis \\ Department of Surgery, Cardiff and Vale NHS Trust, Cardiff CF14 4AX, UK
}

Studies have shown that optimal fluid administration is essential in post-operative surgical management ${ }^{(1,2)}$. Oedema is associated with delayed wound healing, cardiac and respiratory complications and delayed gastrointestinal motility. Problems with the management of fluid balance were highlighted in a survey ${ }^{(3)}$, which concluded that often peri-operative fluid management was suboptimal in the UK.

The aim of the present study was to compare the fluid management of patients who received early enteral nutrition (EEN) or conventional $(\mathrm{CON})$ post-operative management, i.e. nil by mouth. Length of hospital stay, post-operative morbidity and mortality, volume of fluid delivered, cumulative fluid balance and development of oedema were recorded, in addition to other endpoints.

A total of 120 patients (median age 64 (range 35-82) years, sixty oesophageal, thirty-three gastric, twenty-eight pancreatic cancer) were recruited across three National Health Service Trusts over a 3-year period. There were two randomisation groups: group A (EEN) received EEN, commenced within $12 \mathrm{~h}$ of leaving the operating theatre; group B $(\mathrm{CON})$ were 'nil by mouth' with hydration maintained with intravenous (IV) fluids, until deemed safe by the operating surgeon to commence oral diet and fluids.

\begin{tabular}{|c|c|c|c|c|}
\hline Summary of results & CON group $(n 55)$ & EEN group $(n 60)$ & Test statistic & $P$ \\
\hline Incidence of oedema $(n)$ & $17(31 \%)$ & $4(6 \%)$ & Chi 12.0 & 0.0001 \\
\hline Mean cumulative fluid balance $(\mathrm{ml})$ in the first post-operative week & +5123 & $+4053 \mathrm{ml}$ & NS & \\
\hline Mean cumulative volume $(\mathrm{ml})$ of EEN delivered in the first post-operative week & 449 & $7086 \mathrm{ml}$ & F 148 & $>0.0001$ \\
\hline Mean cumulative volume (ml) of IV fluids prescribed in first week & 19574 & 13425 & F 767 & $>0.0001$ \\
\hline Mean level of $\mathrm{Na}(\mathrm{mmol})$ infused via IV fluids & $308 \mathrm{mmol}$ & 154 & NS & \\
\hline Level of $\mathrm{Na}(\mathrm{mmol})$ infused via enteral nutrition & 4 & 35 & NS & \\
\hline Anastomotic leak (\%) & $14.5(n$ 26) & $1.6(n 1)$ & 7.1 & 0.01 \\
\hline Wound infection $(\%)$ & $29(n 16)$ & $5(n 3)$ & 13.1 & 0.001 \\
\hline Patients who opened bowels in first week (\%) & $46.5(n 26)$ & $59(n 35)$ & NS & \\
\hline
\end{tabular}

There were no significant differences in serum albumin concentration between the two groups. The incidence of oedema was higher in the CON group. This was not surprising as the CON group had a higher mean cumulative fluid balance than the EEN group. However, the difference was not sufficient to reflect the marked differences. It may be that the EEN modulated the inflammatory response and affected the rennin-angiotensin II system ${ }^{(4)}$ to modify fluid handling in the EEN group.

To conclude, EEN patients had improved fluid balance when compared with the CON group, this was reflected by a reduction in oedema, improved gastrointestinal transit and fewer complications in the EEN group.

1. Holte K, Sharrock N \& Kehlet H (2002) Br J Anaesth 89, 622-632.

2. Lobo DN (2001) Clin Nutr 20, 125-130.

3. Lobo DN (2002) Ann $R$ Coll Surg Engl 84, 156-160.

4. Desborough J (2000) Br J Anaesth 85, 109-117. 